\title{
Follow-up and management of serologically active clinically quiescent cases in pediatric systemic lupus erythematosus
}

\author{
Gabrielle Capone*, Caroline Lojacono*, Fatma Al-Bayitee ID , Shayan Makvandi, Teresa Hennon, \\ Brian Wrotniak, Rabheh Abdul-Aziz ID \\ ${ }^{*}$ Co-first authors \\ Department of Pediatric Rheumatology, University at Buffalo, United States
}

\begin{abstract}
Objectives: Our aim is to identify the presence of serologically active clinically quiescent (SACQ) episodes in pediatric systemic lupus erythematosus (SLE) patients. We aim to identify serologic biomarkers associated with SACQ episodes and discuss risks and benefits of escalating treatments. Material and methods: We evaluated 25 pediatric SLE patients, 13 of whom experienced SACQ episodes. Serologically active clinically quiescent was defined as two consecutive clinic visits without any clinical symptoms or clinical examination findings of a lupus flare with a clinical Systemic Lupus Erythematosus Disease Activity Index 2000 (SLEDAI-2K) score of zero, but either elevated anti-dsDNA antibodies or low complement (C3 and/or C4) levels.

Results: Among the 13 patients who experienced a SACQ episode, there were a total of 24 episodes, with each patient experiencing 1-4 SACQ episodes. Erythrocyte sedimentation rate (ESR) was the most commonly elevated laboratory marker in a SACQ episode, followed by low hemoglobin levels, and then elevated anti-dsDNA antibodies. Of the 17 episodes treated during a SACQ episode, $15(88 \%)$ did not progress to a clinical flare within six months, while two did. Furthermore, of the 7 patients who were not treated during their SACQ episode, 2 (29\%) continued to be SACQ without flare, whereas 5 led to a clinical flare within six months.

Conclusions: Serologically active clinically quiescent episodes were identified in pediatric SLE patients, suggesting that the presence of SACQ is not limited to adults with SLE. Serologic markers such as increased ESR, hemoglobin, and elevated anti-dsDNA antibodies are preliminarily associated with pediatric SACQ episodes. Treating these SACQ episodes in pediatric SLE patients was less likely to lead to a clinical flare within six months when compared to not treating $(p<0.05)$. More research with a larger sample size is needed to define SACQ episodes, determine the prevalence in pediatric SLE patients, and establish SACQ treatment guidelines.
\end{abstract}

Key words: systemic lupus erythematosus, serologically active clinically quiescent, Systemic Lupus Erythematosus Disease Activity Index 2000.

\section{Introduction}

Systemic lupus erythematosus (SLE) is a chronic autoimmune disease characterized by a variety of clinical manifestations and serologic changes, classically following an undulating course of activity and quiescence, often resulting in multisystem organ damage [1].

In 1979 Gladman et al. [2] identified a subset of adult SLE patients who presented with serologic abnormali- ties in the absence of clinical symptoms. This finding has been subsequently described in approximately $2-15 \%$ of SLE patients [3]. There is ongoing research regarding the efficacy of treatment for these serologically active and clinically quiescent (SACQ) patients [4-6].

This patient population requires a tailored approach to management because treatment protocols should depend on the existence of pathological consequences of the SACQ episodes.

Address for correspondence:

Rabheh Abdul-Aziz, Pediatric Rheumatology, University at Buffalo, 1001 Main Street, 5th Floor, Buffalo, NY 14203, United States, e-mail: raziz@upa.chob.edu

Submitted: 05.04.2021; Accepted: 27.07.2021 
Treatment protocols for SLE are aimed at alleviating symptoms and controlling inflammation, with the overall goal of maintaining quality of life, while avoiding long term organ damage.

Treating patients without clinical symptoms or examination findings risks the morbidity of immunosuppressant and steroid treatment without the benefit of relieving symptoms or the knowledge that the treatment will provide long term benefits [5].

These considerations are especially pertinent in patients with pediatric-onset SLE. An estimated 10-20\% of SLE patients are under the age of 18 [7]. Pediatric-onset SLE patients have a greater risk for renal, central nervous system (CNS), and hematologic manifestations, as well as more organ damage than adult patients [4, 7-10].

Pediatric patients are also susceptible to the negative effects of corticosteroids including but not limited to growth retardation, accelerated atherosclerosis, and severe infectious complications [11].

The presence of SACQ in pediatric-onset SLE has not been identified, even though pediatric SLE patients are at risk of both a more severe disease course and of greater pharmacological morbidities [4, 7, 8, 10].

Gensous et al. [12] concluded in their review, "Since the 1970s, no investigators have succeeded in identifying a biomarker with the potential to predict efficiently the occurrence of new flares, despite great clinical necessity".

The primary aim of this study is to determine the presence of SACQ in pediatric patients with SLE. The secondary aim is to highlight the outcome of escalating or not escalating SLE treatment in the case of SACQ, based on serologic markers such as hypo-complementemia, elevated erythrocyte sedimentation rate (ESR), elevated C-reactive protein (CRP), abnormal platelets, abnormal hemoglobin, and positive anti-dsDNA antibody levels.

\section{Material and methods}

This study protocol was approved by the University at Buffalo Institutional Review Board (IRB). The Institutional Review Board approved exemption from informed consent, as this is a retrospective chart review study. Patients were included in this study if they were followed up at John R. Oishei Children's Hospital in Buffalo, New York between January 2012 and June 2020 and met the revised 1997 American College of Rheumatology (ACR) criteria for systemic lupus erythematosus [13].

Patients were identified by diagnostic codes of systemic lupus erythematosus (ICD-10 code M32.9). Serologically active and clinically quiescent was defined as two consecutive clinic visits without clinical symptoms or examination findings indicative of a lupus flare with a clinical Systemic Lupus Erythematosus Disease Activity Index 2000 (SLEDAI-2K) score of zero, but abnormal blood work, defined by increased anti-dsDNA antibody levels (normal 0-35 $\mathrm{IU} / \mathrm{ml}$ ) and/or low complement levels of C3 (normal range 80-175 mg/dl) or C4 (normal range $14-40 \mathrm{mg} / \mathrm{dl}$ ).

Pediatric SLE patients in our study were typically seen in the clinic every 1-3 months. Twenty-five patients were included in our study: one male and twenty-four females between the ages of five and twenty years old at the time of diagnosis, with disease characteristics as described in Table I.

Thirteen of these patients had episodes that fit our definition of SACQ. We analyzed the medical charts retrospectively, using PowerChart. Data were entered into an electronic database and patient identifications were stored using an anonymous code. We recorded the patients' demographics, clinical presentation at time of diagnosis, initial treatment, and initial blood work results at time of diagnosis (Table I).

Additionally, we recorded medications before and after SACQ periods, along with the blood tests results, which included white blood cells count (WBC), hemoglobin, platelets count, C3 and C4 complement components, antidsDNA antibodies, red blood cells count (RBC) in urine, protein in urine, ESR, CRP, blood urea nitrogen (BUN), creatinine, and other positive antibodies when available. If a patient experienced a clinical flare of SLE following a SACQ episode, its duration in months was recorded.

We used Fisher's exact test to determine whether each of our blood work markers was associated with a SACQ episode. We also used Fisher's exact test to determine whether treatment was associated with fewer clinical flares in six months compared to no treatment. Statistical analyses were based on an alpha of 0.05 and conducted with SYSTAT 13 (SYSTAT Software, 2004).

\section{Results}

Of the 25 pediatric SLE patients evaluated, 13 (52\%) experienced SACQ episodes over the course of the period studied and were included in the study. The mean age at diagnosis of SLE was 14 years. Of the 13 patients who experienced a SACQ episode, the number of episodes of SACQ per patient ranged from 1 to 4 . Seven patients had one SACQ episode, three patients had two episodes, one patient had three episodes, and two patients had four episodes during the period studied. Among the 13 patients, there were a total of 24 SACQ episodes. The percentage frequency of abnormal laboratory values at the beginning of patients' SACQ episodes is displayed in Figure 1. 
Table I. Demographic and disease characteristics of 25 patients with systemic lupus erythematosus

\begin{tabular}{|c|c|}
\hline Parameters & $N=25$ \\
\hline Gender, male/female & $1 / 24$ \\
\hline Age, years & $5-20$ \\
\hline \multicolumn{2}{|l|}{ Race } \\
\hline Caucasian & 10 \\
\hline African American & 11 \\
\hline Asian & 2 \\
\hline Declined & 2 \\
\hline SLE duration in years at time of study & $1-9$ \\
\hline \multicolumn{2}{|l|}{ Symptoms on presentation } \\
\hline Rash & 9 \\
\hline Arthritis & 10 \\
\hline Vasculitis & 1 \\
\hline Oral ulcer & 2 \\
\hline Seizure & 3 \\
\hline Lymphadenopathy & 4 \\
\hline Fatigue & 3 \\
\hline Lupus nephritis & 3 \\
\hline Weight loss & 6 \\
\hline Fever & 8 \\
\hline Arthralgia & 8 \\
\hline Chest pain & 2 \\
\hline Raynaud's phenomena & 1 \\
\hline \multicolumn{2}{|l|}{ Serology on presentation } \\
\hline Low WBC & 8 \\
\hline Anemia & 14 \\
\hline
\end{tabular}

\begin{tabular}{|c|c|}
\hline Parameters & $N=25$ \\
\hline Low platelet & 2 \\
\hline Low C3 & 17 \\
\hline Low C4 & 18 \\
\hline Positive dsDNA & 17 \\
\hline Elevated ESR & 15 \\
\hline Elevated CRP & 4 \\
\hline Proteinuria & 14 \\
\hline Positive ANA & 24 \\
\hline Positive ENA RNP & 13 \\
\hline Positive ENA smith & 11 \\
\hline Positive ENA SSA & 8 \\
\hline Positive ENA SSB & 5 \\
\hline Positive histone antibodies & 6 \\
\hline Positive antiphospholipid & 5 \\
\hline \multicolumn{2}{|c|}{ Treatment in first 6 months of presentation } \\
\hline Steroids & 25 \\
\hline Hydroxychloroquine & 25 \\
\hline Mycophenolate mofetil & 10 \\
\hline Belimumab & 2 \\
\hline Methotrexate & 3 \\
\hline Azathioprine & 2 \\
\hline Cyclophosphamide & 1 \\
\hline Hydrocortisone cream & 3 \\
\hline Anticonvulsant & 3 \\
\hline Anticoagulant & 1 \\
\hline
\end{tabular}

ANA - antinuclear antibodies, CRP - C-reactive protein, ESR - erythrocyte sedimentation rate, SLE - systemic lupus erythematosus, $W B C-$ white blood cells.

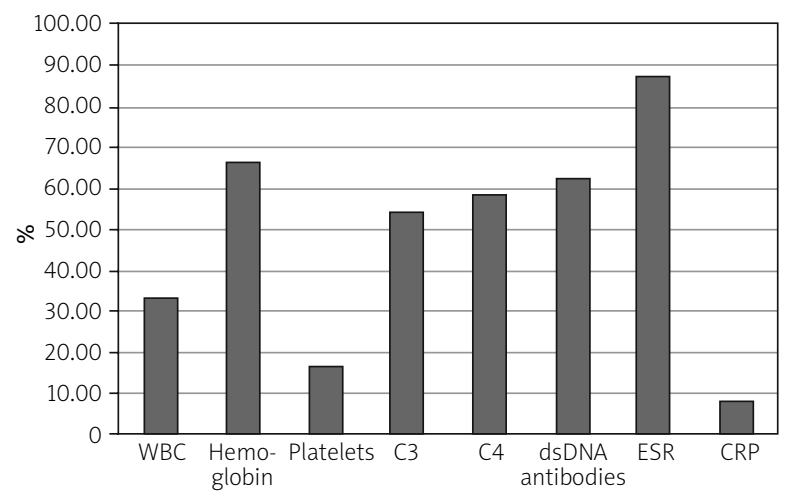

Fig. 1. Percentage frequency of abnormal laboratory values in serologically active and clinically quiescent episodes $(n=24)$.
The laboratory values most commonly found to be abnormal during the 24 SACQ episodes were ESR (87.5\%), hemoglobin (66.6\%), and anti-dsDNA antibodies (62.5\%). None of the laboratory values studied were statistically significantly different between patients who progressed to a clinical flare and those who did not.

Of the 24 episodes of SACQ, 17 (71\%) were treated based on physician's opinion and 7 (29\%) were not treated. Of the 17 episodes treated during a SACQ episode, 15 did not progress to a clinical flare within six months, while two did.

Furthermore, of the 7 patients who were not treated during their SACQ episode, 5 experienced a clinical flare within six months, whereas 2 continued to be SACQ without flare (Table II). 
Table II. Progression to clinical flare among serologically active and clinically quiescent (SACQ) episodes treated versus those not treated

\begin{tabular}{|lccc|}
\hline $\begin{array}{l}\text { Progression to clinical } \\
\text { flare }\end{array}$ & $\begin{array}{c}\text { SACQ and continued to be } \\
\text { SACQ at least 6 months }\end{array}$ & $\begin{array}{c}\text { SACQ leads to clinical } \\
\text { symptoms }\end{array}$ & Marginin 6 months row totals \\
\hline With treatment & 15 & 2 & 17 \\
\hline Without treatment & 2 & 5 & 7 \\
\hline Total & 17 & 7 & 24 (grand total) \\
\hline
\end{tabular}

Results indicated a lower percentage progression to clinical flare for serologically active and clinically quiescent (SACQ) episodes treated, with a prevalence of $12 \%(2 / 17)$, compared to $71 \%(5 / 7)$ progression to clinical flare when not treated $(p<0.05)$.

Treating the SACQ episodes was less likely to lead to a clinical flare within six months when compared to not treating a SACQ $(p<0.05)$. Table III shows a full description of SACQ episodes.

\section{Discussion}

\section{Definition of serologically active and clinically quiescent}

To our knowledge, this is the first study evaluating SACQ episodes in the pediatric SLE population; all studies we are aware of were conducted with adult-onset SLE patients [2, 3, 5, 14-18].

The primary aim of this study was to evaluate for the presence of SACQ episodes in pediatric-onset SLE patients, and to determine whether treatment of these episodes may be beneficial in preventing the onset of a clinical flare. During the period studied, 13/25 patients (52\%) with pediatric-onset SLE experienced a SACQ episode. This is a significantly higher frequency than has been described in the literature evaluating the adult SLE population, with most describing a prevalence between 2.2 and $15 \%[14,15,17,18]$.

This is likely due to various factors. First and foremost, there are variations in the definition of a SACQ episode. Many adult SLE studies have defined a SACQ episode as two or more consecutive years without clinical symptoms $[3,5,14,16]$.

Our definition required two consecutive clinic visits without clinical symptoms or examination findings indicative of a lupus flare, and in most cases this would equate to 4-6 months. As discussed previously, pediatric-onset SLE patients have a greater risk for renal, CNS, and hematologic manifestations, and most importantly organ damage. For this reason, pediatric SLE patients are monitored more frequently with both laboratory and clinical assessments.

A second variation in the definition is in the laboratory criteria. Ng et al. [18] defined SACQ as a mean global BILAG (British Isles Lupus Assessment Group) score less than 6 and anti-dsDNA antibody titers above the normal level on at least 2 occasions during a 6 -month period.
In our study, SACQ was defined by elevated anti-dsDNA antibodies and/or low C3 or C4 levels. This definition was chosen based on both the literature [6] and the personal experience of the treating physicians that decreases in complement levels and increases in anti-dsDNA antibodies often herald the onset of a clinical flare.

Unfortunately, potentially due to the small sample size in our study, neither abnormal anti-dsDNA antibody levels nor C3 or C4 levels differentiated clinical flares. It is worth mentioning, however, that abnormal anti-dsDNA antibody levels were seen in 62.5\% $(n=15)$ of SACQ episodes, $53 \%(n=8)$ of which went on to clinical flare compared to $22 \%(n=2)$ of normal anti-dsDNA antibody episodes which continued on to clinical flare.

A second difference in methodology between previous adult SLE studies and our study is that we included patients taking corticosteroids and/or immunosuppressive medications. These patients have been excluded in some previous adult studies because this could be a confounding factor in normalizing blood work [3, 5, $14,16]$. Lastly, our study consisted of a small sample size $(n=25)$, with only $13(52 \%)$ out of the 25 patients experiencing a SACQ episode, limiting statistical power and extrapolation.

\section{Development of serologically active and clinically quiescent into clinical flare}

There has been research investigating the prevalence of clinical flares developing from SACQ episodes.

Walz LeBlanc et al. [15] observed that almost half of the 74 patients with SLE who had a SACQ period experienced a flare within a year, using the SLEDAI global activity score, with no predictive factors identified during or before the SACQ period.

Steiman et al. [14] reported that a similar proportion (58.9\%) of patients who experienced a SACQ episode developed a clinical flare at a longer median of 158 weeks.

In a more long term study, $\mathrm{Ng}$ et al. [18] found that $9 \%(n=27)$ of 290 SLE patients had SACQ episodes, with 17 (81\%) SACQ episodes leading to a flare in the next five years. Median duration to the first flare was 15 months (range 2-46 months). They also suggested that anti- 


\begin{tabular}{|c|c|c|c|c|c|c|c|c|c|c|c|c|}
\hline 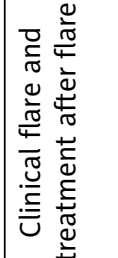 & 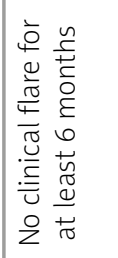 & 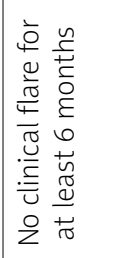 & 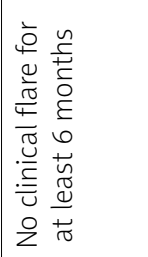 & 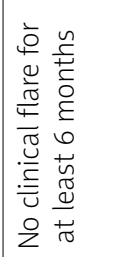 & 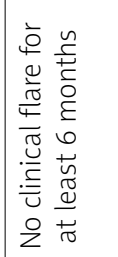 & 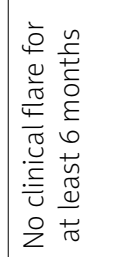 & 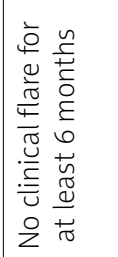 & 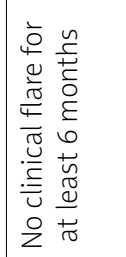 & 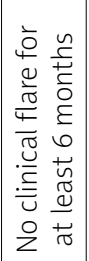 & 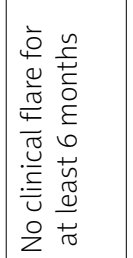 & 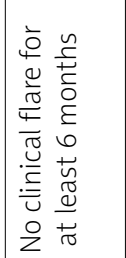 & 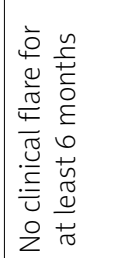 \\
\hline 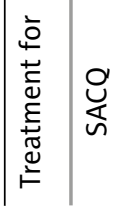 & 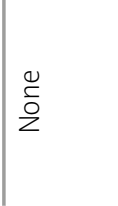 & 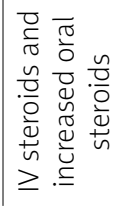 & 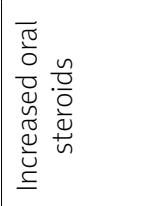 & 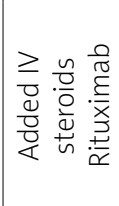 & $\begin{array}{l}\frac{n}{0} \\
\frac{0}{0} \\
\frac{0}{4} \\
4 \\
\geq \\
\frac{7}{0} \\
\frac{0}{4}\end{array}$ & 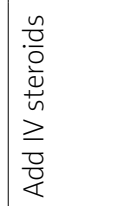 & 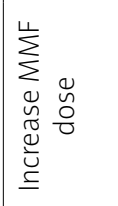 & $\begin{array}{l}\frac{n}{0} \\
\frac{0}{2} \\
\frac{0}{4} \\
\geq \\
\frac{0}{0} \\
\frac{0}{4}\end{array}$ & 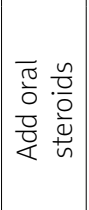 & 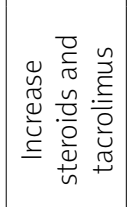 & 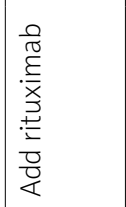 & 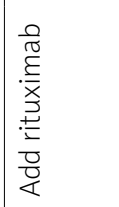 \\
\hline 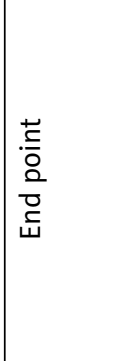 & 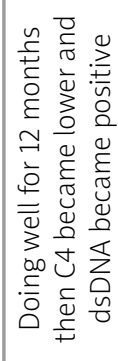 & 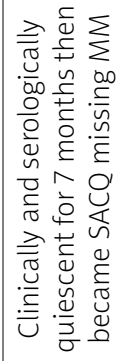 & 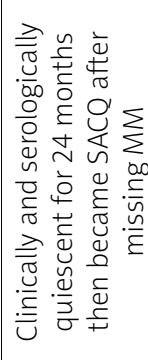 & 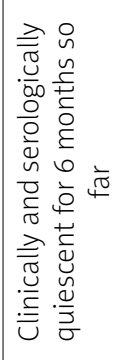 & 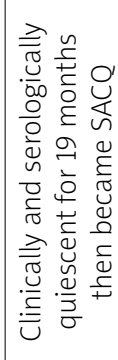 & 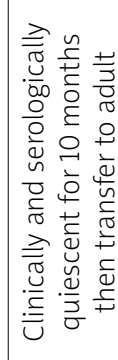 & 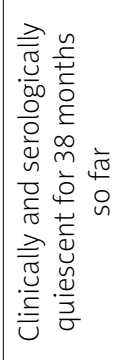 & 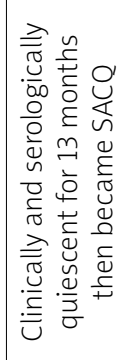 & 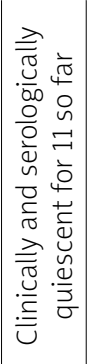 & 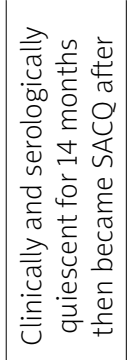 & 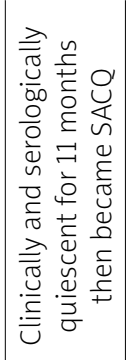 & 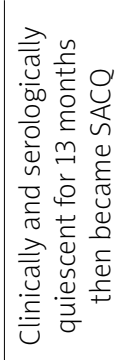 \\
\hline 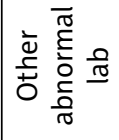 & 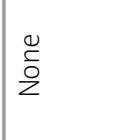 & $\begin{array}{l}\frac{0}{2} \\
2 \\
2\end{array}$ & 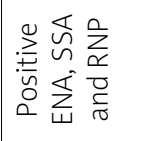 & 总 & $\begin{array}{l}\frac{\pi}{0} \\
\stackrel{0}{2}\end{array}$ & $\begin{array}{l}0 \\
\stackrel{0}{0} \\
2\end{array}$ & 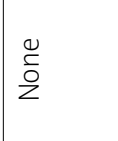 & $\begin{array}{l}\frac{\pi}{0} \\
\stackrel{0}{2}\end{array}$ & 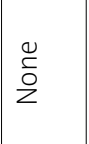 & 号 & 号 & 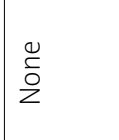 \\
\hline 穵 & $\stackrel{\sim}{0}$ & $\stackrel{\sim}{0}$ & $\stackrel{\sim}{\circ}$ & m. & $\stackrel{0}{\circ}$ & $\begin{array}{l}0 \\
0\end{array}$ & $\stackrel{\overbrace{}}{\longrightarrow}$ & $\rightrightarrows$ & 9 & $\sim$ & 6 & $\stackrel{0}{0}$ \\
\hline 总 & $\stackrel{\grave{m}}{ }$ & ๆ & $\stackrel{\circ}{\sim}$ & in & $\stackrel{\infty}{\sim}$ & $\ddot{\sim}$ & $\approx$ & 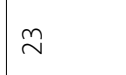 & $\stackrel{\llcorner}{\infty}$ & $\widetilde{\sigma}$ & 尺 & ๙ \\
\hline 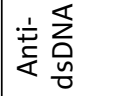 & $\begin{array}{l}\infty \\
\stackrel{\infty}{2}\end{array}$ & $\stackrel{\stackrel{\bullet}{m}}{\stackrel{m}{m}}$ & $\overrightarrow{\vec{n}}$ & $\underline{\underline{\sigma}}$ & $\frac{\infty}{20}$ & $\stackrel{\infty}{2}$ & $\begin{array}{l}\infty \\
\stackrel{\infty}{\sim}\end{array}$ & $\vec{\sigma}$ & ৪্ & $\stackrel{\infty}{2}$ & $\begin{array}{l}\infty \\
\stackrel{\infty}{Z}\end{array}$ & $\begin{array}{l}\infty \\
\stackrel{\infty}{z}\end{array}$ \\
\hline U & $\stackrel{+}{\circ}$ & $\stackrel{\overbrace{}}{-}$ & ナ & $m$ & $\exists$ & $\approx$ & ñ. & $\stackrel{\sim}{\sim}$ & $\stackrel{\curvearrowright}{\sim}$ & $\approx$ & 0 & $\stackrel{\searrow}{\sim}$ \\
\hline 0 & $\hat{0}$ & $\stackrel{+}{\sim}$ & fo & $\vec{F}$ & 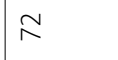 & $\tilde{\theta}$ & $\hat{a}$ & $\stackrel{\infty}{+}$ & $\vec{\sigma}$ & $\begin{array}{l}\infty \\
\infty\end{array}$ & $\stackrel{\infty}{\wedge}$ & 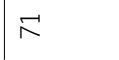 \\
\hline$\frac{\pi}{2}$ & $\stackrel{\circ}{\stackrel{0}{m}}$ & $\stackrel{\infty}{\stackrel{\infty}{\sim}}$ & 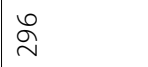 & $m$ & $\stackrel{\circ}{\circ}$ & $\stackrel{n}{\stackrel{n}{n}}$ & $\vec{m}$ & $\stackrel{n}{\sim}$ & $\stackrel{m}{\infty}$ & $\stackrel{n}{m}$ & ๑ & 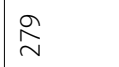 \\
\hline 오 & $\begin{array}{l}0 \\
\stackrel{9}{9}\end{array}$ & $\stackrel{\AA}{\nexists}$ & $\Rightarrow$ & $\stackrel{m}{\stackrel{\rho}{\rho}}$ & $\stackrel{\infty}{\sigma}$ & $\begin{array}{l}m \\
0 \\
0\end{array}$ & $\begin{array}{l}\infty \\
\underset{\sim}{\sim}\end{array}$ & $\stackrel{\stackrel{n}{G}}{\exists}$ & m. & $\vec{m}$ & $\underset{\Im}{\stackrel{\sim}{\sim}}$ & $\stackrel{m}{=}$ \\
\hline 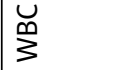 & ڤึ & $\vec{F}$ & in & $\stackrel{\sim}{\underset{f}{f}}$ & t & $\underset{\leftarrow}{\mathscr{\leftarrow}}$ & m & $\stackrel{o}{\sim}$ & $\stackrel{\text { aे }}{\text { in }}$ & $\begin{array}{l}\infty \\
0 \\
0\end{array}$ & $\stackrel{\sim}{\wedge}$ & $\hat{i}$ \\
\hline 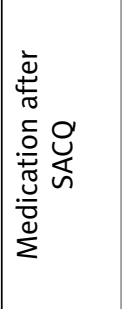 & $\begin{array}{l}\stackrel{\Xi}{E} \\
\sim \\
\sim\end{array}$ & 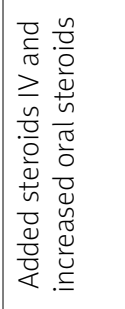 & 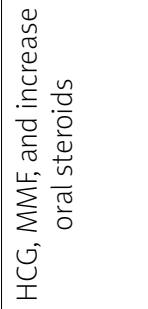 & 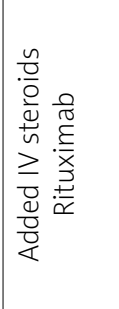 & $\begin{array}{l}\frac{n}{0} \\
\frac{0}{0} \\
\frac{0}{4} \\
2 \\
\frac{7}{2} \\
\frac{\pi}{4}\end{array}$ & $\frac{\geq}{\frac{n}{0}}$ & 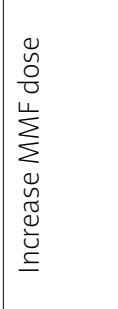 & 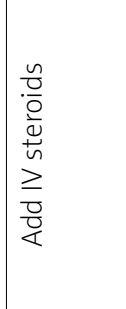 & 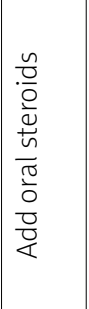 & 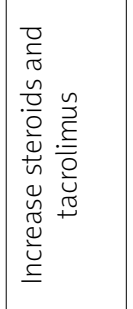 & 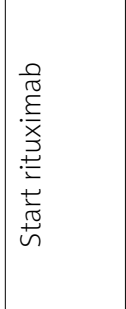 & 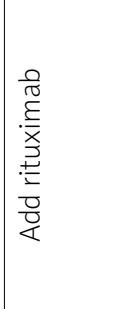 \\
\hline 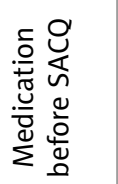 & 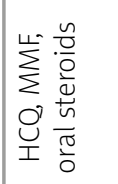 & 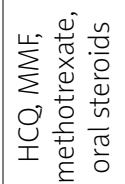 & 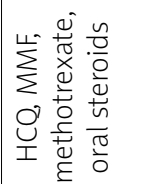 & 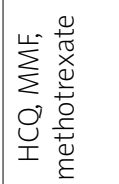 & 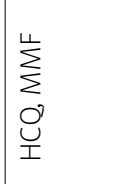 & $\begin{array}{l}\sum_{\sum}^{u} \\
0 \\
0 \\
\stackrel{U}{1}\end{array}$ & 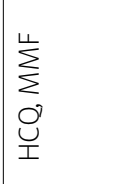 & $\begin{array}{l}\sum_{\sum}^{u} \\
0 \\
\stackrel{U}{1} \\
\end{array}$ & 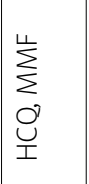 & 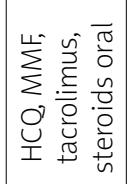 & 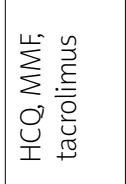 & 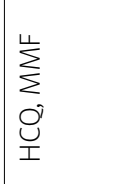 \\
\hline 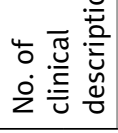 & 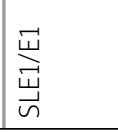 & 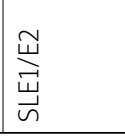 & 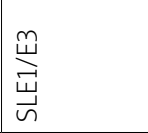 & 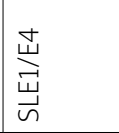 & 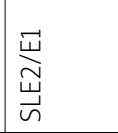 & 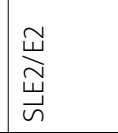 & $\frac{\ddot{m}}{\sim}$ & 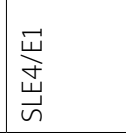 & \begin{tabular}{|l}
$\frac{\tilde{u}}{J}$ \\
$\vec{山}$ \\
$\vec{\omega}$
\end{tabular} & 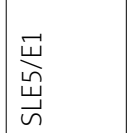 & 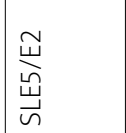 & 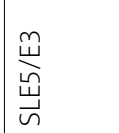 \\
\hline
\end{tabular}




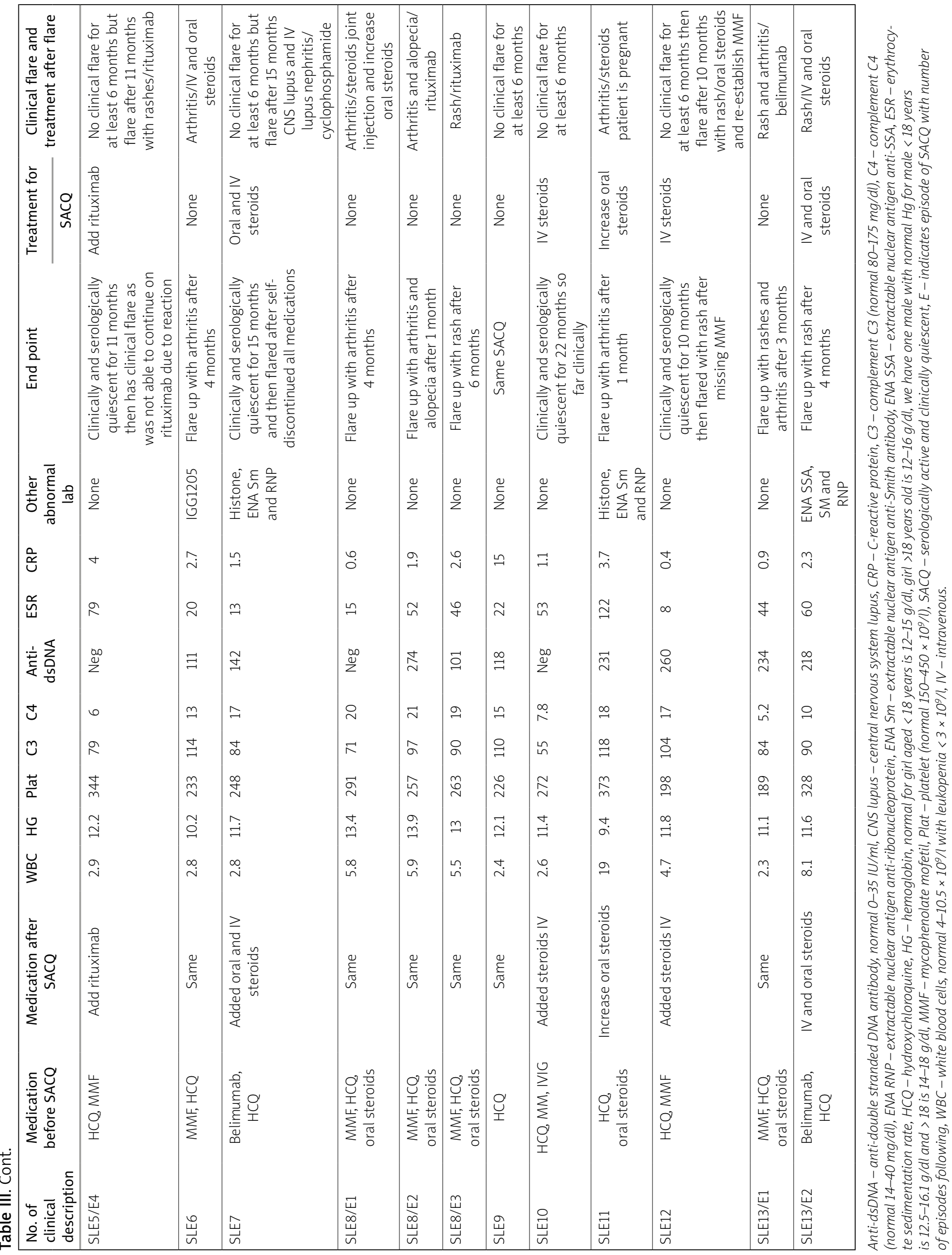


nucleosome antibodies (anti-NCS) may be a better predictor than anti-dsDNA antibodies for future flares.

Steiman et al. [5] observed that patients with a prolonged SACQ period accrued less damage over a decade compared to matched (SLE) controls, supporting a conservative treatment approach.

This literature has supported the general consensus that active serology without clinical manifestations should not guide treatment decisions, and that these patients are best managed conservatively with close follow-up.

However, this is not always an approach that physicians are comfortable with. For example, one patient in our study presented with severe manifestations at the time of diagnosis, including seizures, thrombosis in multiple organs, and an eleven year history of lymphadenopathy; this was previously published as a case report [19].

Following treatment he went into remission both clinically and serologically. In a follow-up visit, he was positive for anti-dsDNA antibodies after having previously tested negative and had elevated ESR after it was previously normal with no clinical symptoms, prompting the physician to advise treatment.

Despite this recommendation, the patient chose not to be treated, and six months later presented to the emergency room with a very severe clinical flare. This is an example of a case where treating a SACQ period may prevent a clinical flare.

\section{Treatment considerations}

One of the goals of our study was to determine whether treating a SACQ episode is associated with a better clinical outcome. According to our data (Table II), $71 \%$ of SACQ flares that were not treated led to clinical flares within 6 months compared to only $12 \%$ of SACQ flares that were treated.

Due to multiple scoring systems for monitoring SLE and following flares, physician's opinion is often considered the 'gold standard' for the evaluation of disease activity with bias based on personal experience [20].

In our study, physician's opinion was used to decide whether or not to escalate treatment of the SACQ episode based on the physician's knowledge of each patient. For example, in our study we have a case of a patient with a SACQ episode with significantly low platelets of 13,000, low C3, low C4, and positive anti-dsDNA antibodies without any clinical sign of bleeding who underwent treatment for low platelets given the balance of benefit and risk of treatment. This patient went into remission serologically and continued to be in remission clinically.

If serological activity serves as a marker of future clinical manifestations, and if underlying damage were to persist even in the absence of symptoms, it would be appropriate to institute a treatment protocol for abnormal serology. However, if SACQ flares neither cause harm nor indicate future harm, the patient risks the morbidity of immunosuppressant or steroid treatment without gaining the benefit of pharmacological intervention.

Treatment of a SACQ episode usually consists of adding either corticosteroids or an immunosuppressant medication. Pediatric-onset SLE patients have a longer disease course and are at greater risk of morbidity from medications. Corticosteroid use can lead to acne, weight gain, cushingoid appearance, growth retardation, accelerated atherosclerosis, and severe infectious complications [11].

Physicians have to weigh the risks and benefits of treatment carefully. Adolescent patients in particular may struggle with medication compliance as they grapple with the severity and chronicity of their diagnosis and the side effects of the medications. In this population, non-adherence to medication regimens is of particular concern and could be associated with SACQ episodes.

Several patients in our study had low or non-detectable medication levels when tested. It is important to establish evidence-based approaches for SACQ episodes so pediatric patients do not experience negative consequences of unnecessary treatment or systemic damage that could have been avoided by treatment.

\section{Conclusions}

Decisions surrounding the benefits and risks of treating SACQ SLE prove even more challenging in the pediatric population due to more severe lupus manifestations, longer disease course, unknown risk of end organ damage in SACQ disease, and unknown data about risk of flares.

The goals of therapy for patients with SLE are to ensure long-term survival, achieve the lowest possible disease activity, prevent organ damage, minimize drug toxicity, improve quality of life, and educate patients and their families about their role in disease management.

The management of disease flares in children with SLE is highly individualized. Further research with prospective study is warranted to determine whether pediatric patients with SACQ episodes should be treated and how these decisions then affect the health of these patients in the long term.

The authors declare no conflict of interest. 


\section{References}

1. Tomioka R, Tani K, Sato K, et al. Observations on the occurrence of exacerbations in clinical course of systemic lupus erythematosus. J Med Invest 2008; 55: 112-119, DOI: 10.2152/ jmi.55.112.

2. Gladman DD, Urowitz MB, Keystone EC. Serologically active clinically quiescent systemic lupus erythematosus: a discordance between clinical and serologic features. Am J Med 1979; 66: 210-215, DOI: 10.1016/0002-9343(79)90529-1.

3. Conti F, Ceccarelli F, Perricone C, et al. Flare, persistently active disease, and serologically active clinically quiescent disease in systemic lupus erythematosus: a 2-year follow-up study. PLoS One 2012; 7: e45934, DOI: 10.1371/journal.pone.0045934.

4. Barron KS, Silverman ED, Gonzales J, Reveille JD. Clinical, serologic, and immunogenetic studies in childhood-onset systemic lupus erythematosus. Arthritis Rheum 1993; 36: 348-354, DOI: $10.1002 /$ art.1780360310.

5. Steiman AJ, Gladman DD, Ibañez D, Urowitz MB. Outcomes in patients with systemic lupus erythematosus with and without a prolonged serologically active clinically quiescent period. Arthritis Care Res (Hoboken) 2012; 64: 511-518, DOI: 10.1002/ acr.21568.

6. Tseng CE, Buyon JP, Kim M, et al. The effect of moderate-dose corticosteroids in preventing severe flares in patients with serologically active, but clinically stable, systemic lupus erythematosus: findings of a prospective, randomized, double-blind, placebo-controlled trial. Arthritis Rheum 2006; 54: 3623-3632, DOI: 10.1002/art.22198.

7. Mina R, Brunner HI. Pediatric lupus - are there differences in presentation, genetics, response to therapy, and damage accrual compared with adult lupus? Rheum Dis Clin North Am 2010; 36: 53-80, vii-viii, DOI: 10.1016/j.rdc.2009.12.012.

8. Font J, Cervera R, Espinosa G, et al. Systemic lupus erythematosus (SLE) in childhood: analysis of clinical and immunological findings in 34 patients and comparison with SLE characteristics in adults. Ann Rheum Dis 1998; 57: 456-459, DOI: 10.1136/ard.57.8.456

9. Rood MJ, ten Cate R, van Suijlekom-Smit LW, et al. Childhood-onset systemic lupus erythematosus: clinical presentation and prognosis in 31 patients. Scand J Rheumatol 1999; 28: 222-226, DOI: 10.1080/03009749950155580.
10. Ravelli A, Duarte-Salazar C, Buratti S, et al. Assessment of damage in juvenile-onset systemic lupus erythematosus: a multicenter cohort study. Arthritis Rheum 2003; 49: 501507, DOI: 10.1002/art.11205.

11. Niaudet P. Treatment of lupus nephritis in children. Pediatr Nephrol 2000; 14: 158-166, DOI: 10.1007/s004670050034.

12. Gensous N, Marti A, Barnetche T, et al. Predictive biological markers of systemic lupus erythematosus flares: a systematic literature review. Arthritis Res Ther 2017; 19: 238, DOI: 10.1186/s13075-017-1442-6.

13. Hochberg MC. Updating the American College of Rheumatology revised criteria for the classification of systemic lupus erythematosus. Arthritis Rheum 1997; 40: 1725, DOI: 10.1002/ art.1780400928.

14. Steiman AJ, Gladman DD, Ibañez D, Urowitz MB. Prolonged serologically active clinically quiescent systemic lupus erythematosus: frequency and outcome. J Rheumatol 2010; 37: 1822-1827, DOI: 10.3899/jrheum.100007.

15. Walz LeBlanc BA, Gladman DD, Urowitz MB. Serologically active clinically quiescent systemic lupus erythematosus - predictors of clinical flares. J Rheumatol 1994; 21: 2239-2241.

16. Steiman AJ, Urowitz MB, Ibañez D, et al. Anti-dsDNA and antichromatin antibody Isotypes in serologically active clinically quiescent systemic lupus erythematosus. J Rheumatol 2015; 42: 810-816, DOI: 10.3899/jrheum.140796.

17. LeBlanc BA, Urowitz MB, Gladman OD. Serologically active, clinically quiescent systemic lupus erythematosus - longterm followup. J Rheumatol 1994; 21: 174-175.

18. Ng KP, Manson JJ, Rahman A, Isenberg DA. Association of antinucleosome antibodies with disease flare in serologically active clinically quiescent patients with systemic lupus erythematosus. Arthritis Rheum 2006; 55: 900-904, DOI: 10.1002/ art.22356.

19. Mull ES, Aranez V, Pierce D, et al. Newly diagnosed systemic lupus erythematosus: atypical presentation with focal seizures and long-standing lymphadenopathy. J Clin Rheumatol 2019; 25: e109-e113, DOI: 10.1097/RHU.0000000000000681.

20. Wollaston SJ, Farewell VT, Isenberg DA, et al. Defining response in systemic lupus erythematosus: a study by the Systemic Lupus International Collaborating Clinics group. J Rheumatol 2004; 31: 2390-2394. 